\title{
INFERENCE ON TWO-COMPONENT MIXTURES UNDER TAIL RESTRICTIONS
}

\author{
KOEN JOCHMANS \\ Sciences PO \\ MaRC HENRY \\ The Pennsylvania State University \\ Bernard Salanié \\ Columbia University
}

\begin{abstract}
Many econometric models can be analyzed as finite mixtures. We focus on twocomponent mixtures, and we show that they are nonparametrically point identified by a combination of an exclusion restriction and tail restrictions. Our identification analysis suggests simple closed-form estimators of the component distributions and mixing proportions, as well as a specification test. We derive their asymptotic properties using results on tail empirical processes and we present a simulation study that documents their finite-sample performance.
\end{abstract}

\section{INTRODUCTION}

The use of finite mixtures has a long history in applied econometrics. A nonexhaustive list of applications includes models with discrete unobserved heterogeneity, hidden Markov chains, and models with mismeasured discrete variables; see Henry, Kitamura, and Salanié (2014) for a more extensive discussion of applications. Until recently, the literature on nonparametric identification of mixture models was sparse. Following the lead of Hall and Zhou (2003), several authors have analyzed multivariate mixtures; recent contributions are Kasahara and Shimotsu (2009), Allman, Matias, and Rhodes (2009), and Bonhomme, Jochmans, and Robin (2014; 2016). There are fewer identifying restrictions available when the model of interest is univariate. Bordes, Mottelet,

\footnotetext{
We are grateful to Peter Phillips, Arthur Lewbel, and three referees for comments and suggestions, and to Victor Chernozhukov and Yuichi Kitamura for fruitful discussions. Parts of this paper were written while Henry was visiting the University of Tokyo Graduate School of Economics and while Salanié was visiting the Toulouse School of Economics. The hospitality of both institutions is gratefully acknowledged. Jochmans' research has received funding from the SAB grant "Nonparametric estimation of finite mixtures". Henry's research has received funding from the SSHRC Grants 410-2010-242 and 435-2013-0292, and NSERC Grant 356491-2013. Salanié thanks the Georges Meyer endowment. Some of the results presented here previously circulated as part of Henry, Kitamura, and Salanié (2010), whose published version (Henry et al., 2014) only contains results on partial identification. Address correspondence to Koen Jochmans, Sciences Po, Department of Economics, 28 rue des Saints Pères 75007, Paris, France, e-mail. koen.jochmans@sciencespo.fr.
} 
and Vandekerkhove (2006), for instance, provide such restrictions for location models with symmetric error distributions.

In this paper, we give sufficient conditions that point-identify univariate component distributions and associated mixing proportions. The restrictions we rely on are most effective in two-component models; and to simplify the analysis, we focus on this case, like Hall and Zhou (2003) and Bordes et al. (2006). We comment briefly on mixtures with more components at the end of the paper. Our arguments are constructive, and we propose closed-form estimators for both the component distributions and the mixing proportions. We derive their large-sample properties, and we propose a specification test. Finally, we investigate the behavior of our inference tools in a simulation experiment.

The model we consider in this paper is characterized by an exclusion restriction and a tail-dominance assumption. Like Henry et al. (2014), we assume the existence of a source of variation that shifts the mixing proportions but leaves the component distributions unchanged. Such an assumption is natural in several important applications, such as measurement-error models (Mahajan, 2006), for example. In hidden Markov models, it follows directly from the model specification. The exclusion restriction is also implied by the conditional-independence restriction that underlies the results of Hall and Zhou (2003) and others on multivariate mixtures.

Henry et al. (2014) have shown that our exclusion restriction implies that both the mixing proportions and the component distributions lie in a nontrivial set. However, they only proved partial identification, and they did not discuss inference. Here, we achieve point-identification by complementing the exclusion restriction with a restriction on the relative tail behavior of the component distributions. This restriction is quite natural in location models, for instance, but it can be motivated more generally. Regime-switching models typically feature regimes with different tail behavior, for example. Alternatively, theoretical models can imply the required tail behavior; an example is the search and matching model of Shimer and Smith (2000), as explained in D'Haultfœuille and Février (2015).

Our identification argument suggests plug-in estimators of the mixing proportions and the component distributions that are available in closed form. The estimators are based on ratios of intermediate quantiles, and their convergence rate is determined by the theory of tail empirical processes. As we rely on the tail behavior of the component distributions to infer the mixing proportions, our estimators converge more slowly than the parametric rate. If the mixing proportions were known - or could be estimated at the parametric rate- the tail restrictions could be dispensed with and the implied estimator of the component distributions would also converge at the parametric rate.

Our estimators are consistent under very weak tail-dominance assumptions. To control for asymptotic bias in their limit distribution, we need to impose stronger requirements that prevent the tails of the components from vanishing too quickly. These assumptions rule out the Gaussian location model. Such thin-tailed distributions are known to be problematic for inference techniques that rely on tail 
behavior (Khan and Tamer, 2010). However, we show that our assumptions apply to distributions with fatter tails, such as Pareto distributions.

Identification only requires that the variable subject to the exclusion restriction can take on two values. If it can take on more values, the model is overidentified and the specification can be tested.

The tail conditions we use to obtain nonparametric identification are related to the well-known identification-at-infinity argument of Heckman (1990); see also D'Haultfœuille and Maurel (2013) for another approach. Other types of support restrictions have been used in related problems to establish identification. Schwarz and Van Bellegem (2010) imposed support restrictions in a semiparametric deconvolution problem to deal with measurement error in location models. D'Haultfœuille and Février (2015) relied on a support condition as an alternative to completeness conditions ( $\mathrm{Hu}$ and Schennach, 2008) in multivariate mixture models.

The remainder of the paper is organized as follows. Section 1 describes the mixture model and proves identification. We rely on these results to construct estimators and derive their asymptotic properties in Section 2. We also discuss specification testing at this point. In Section 3, we conduct a Monte Carlo experiment that gives evidence on the small-sample performance of our methods. Finally, we conclude with some remarks on mixtures with more than two components.

\section{MIXTURES WITH EXCLUSION AND TAIL RESTRICTIONS}

Let $(Y, X) \in \mathbb{R} \times \mathcal{X}$ be random variables. We assume throughout that our mixtures satisfy the following simple exclusion restriction. ${ }^{1}$

Assumption 1 (Mixture with exclusion). $F(y \mid x) \equiv \mathbb{P}(Y \leq y \mid X=x)$ decomposes as the two-component mixture

$$
F(y \mid x)=G(y) \lambda(x)+H(y)(1-\lambda(x))
$$

for distribution functions $G: \mathbb{R} \mapsto[0,1]$ and $H: \mathbb{R} \mapsto[0,1]$ and a function $\lambda$ : $\mathcal{X} \mapsto[0,1]$ that maps values $x$ into mixing proportions.

The assumption that the component distributions do not depend on $X$ embodies our exclusion restriction; see also Henry et al. (2014).

We complete the mixture model with the following assumption.

Assumption 2. The mixing proportion $\lambda$ is nonconstant on $\mathcal{X}$ and is bounded away from zero and one on $\mathcal{X}$.

Nonconstancy of $\lambda$ gives the variable $X$ relevance. Bounding $\lambda$ away from zero and one implies that the mixture is irreducible. ${ }^{2}$

\subsection{Motivating examples}

Our first example has a long history in empirical work (Frisch, 1934). 
Example 1 (Mismeasured treatments)

Let $T$ denote a binary treatment indicator. Suppose that $T$ is subject to classification error: rather than observing $T$, we observe misclassified treatment $X$. The distribution of the outcome variable $Y$ given $X=x$ is

$$
F(y \mid x)=\mathbb{P}(Y \leq y \mid T=1, X=x) \lambda(x)+\mathbb{P}(Y \leq y \mid T=0, X=x)(1-\lambda(x)),
$$

with $\lambda(x)=\mathbb{P}(T=1 \mid X=x)$. The usual ignorability assumption states that $X$ and $Y$ are independent given $T$. That is,

$\mathbb{P}(Y \leq y \mid T=t, X=x)=\mathbb{P}(Y \leq y \mid T=t)$,

for $t \in\{0,1\}$, in which case the decomposition of $F(y \mid x)$ reduces to the model in (1.1) with $G(y)=\mathbb{P}(Y \leq y \mid T=1)$ and $H(y)=\mathbb{P}(Y \leq y \mid T=0)$. Also note that $\lambda$ is nonconstant unless misclassification in $T$ is completely random.

The identification of treatment effects when the treatment indicator is mismeasured has received considerable attention, especially in the context of regression models (Bollinger, 1996; Mahajan, 2006; Lewbel, 2007). Here, the conditional ignorability assumption that validates our exclusion restriction relies on nondifferential misclassification error. It has been routinely used elsewhere (Carroll, Ruppert, Stefanski, and Crainiceanu, 2006).

Our second example deals with regime-switching models, also referred to as hidden Markov models. These models cover switching regressions, which have been used in a variety of settings (see, e.g., Heckman, 1974, Hamilton, 1989), as well as several versions of stochastic-volatility models (Ghysels, Harvey, and Renault, 1996).

\section{Example 2 (Hidden Markov model)}

Let $\boldsymbol{Y}=\left(Y_{1}, \ldots, Y_{T}\right)^{\prime}$ be a time series of outcome variables. A hidden Markov model for the dependency structure in these data assumes that there is a discrete latent series of state variables $\boldsymbol{S}=\left(S_{1}, \ldots, S_{T}\right)^{\prime}$ having Markovian dependence, so that the variables in $\boldsymbol{Y}$ are jointly independent given $\boldsymbol{S}$, and that

$\mathbb{P}\left(Y_{t} \leq y_{t} \mid \boldsymbol{S}=s\right)=\mathbb{P}\left(Y_{t} \leq y_{t} \mid S_{t}=s_{t}\right)$.

To see that such a model fits (1.1), assume that there are two latent states 0 and 1 and (for notational simplicity) that $\boldsymbol{S}$ has first-order Markov dependence. Denote $\boldsymbol{X}=\left(Y_{1}, \ldots, Y_{t-1}\right)^{\prime}$. Then

$F\left(y_{t} \mid \boldsymbol{x}\right)=\mathbb{P}\left(Y_{t} \leq y_{t} \mid S_{t}=1\right) \mathbb{P}\left(S_{t}=1 \mid \boldsymbol{X}=\boldsymbol{x}\right)+\mathbb{P}\left(Y_{t} \leq y_{t} \mid S_{t}=0\right) \mathbb{P}\left(S_{t}=0 \mid \boldsymbol{X}=\boldsymbol{x}\right)$,

which fits our setup. Moreover, $\lambda(\boldsymbol{x})=\mathbb{P}\left(S_{t}=1 \mid \boldsymbol{X}=\boldsymbol{x}\right)$ does vary with $\boldsymbol{x}$, unless the outcomes are independent of the latent states.

In this example, the exclusion restriction follows directly from the Markovian structure of the regime-switching model. Gassiat and Rousseau (2016) obtained 
nonparametric identification in location models when the matrix of transition probabilities of the Markov chain has full rank. The approach presented here delivers nonparametric identification in a much broader range of models.

Our third example links (1.1) to the recent literature on multivariate mixtures that builds on Hall and Zhou (2003).

Example 3 (Multivariate mixture)

Suppose $Y$ and $X$ are two measurements that are independent conditional on a latent binary factor $T$ :

$$
\begin{aligned}
\mathbb{P}(Y \leq y, X \leq x) & =\mathbb{P}(Y \leq y \mid T=1) \mathbb{P}(X \leq x \mid T=1) \mathbb{P}(T=1) \\
& +\mathbb{P}(Y \leq y \mid T=0) \mathbb{P}(X \leq x \mid T=0) \mathbb{P}(T=0) .
\end{aligned}
$$

Then the conditional distribution of the $Y$ given $X$ is

$$
F(y \mid x)=\mathbb{P}(Y \leq y \mid T=1) \mathbb{P}(T=1 \mid X=x)+\mathbb{P}(Y \leq y \mid T=0) \mathbb{P}(T=0 \mid X=x) .
$$

This is of the form in (1.1) with $G(y)=\mathbb{P}(Y \leq y \mid T=1), H(y)=\mathbb{P}(Y \leq y \mid T=0)$, and $\lambda(x)=\mathbb{P}(T=1 \mid X=x)$. Note that the bivariate mixture model implies that the distribution of $X$ given $Y$ decomposes in the same way.

Hall and Zhou (2003) showed that multivariate two-component mixtures with conditional-independence restrictions are nonparametrically identified from data on three or more measurements and are set identified from data on only two measurements. The results we derive below imply that two measurements can yield point identification under tail restrictions.

\subsection{Identification}

We show below that both the mixture components $G, H$ and the mixing proportions $\lambda$ are identified under the following dominance condition on the tails of the component distributions.

Assumption 3 (Tail dominance).

(i) The left tail of $G$ is thinner than the left tail of $H$, i.e.,

$$
\lim _{y \downarrow-\infty} \frac{G(y)}{H(y)}=0 .
$$

(ii) The right tail of $G$ is thicker than the right tail of $H$, i.e.,

$$
\lim _{y \uparrow+\infty} \frac{1-H(y)}{1-G(y)}=0 .
$$

Tail dominance is natural in location models. 
Example 4 (Location models)

Suppose that $Y=\mu(T)+U$, where $T$ is a binary indicator and $U \sim F$, independent of $T$. Then (1.1) yields

$F(y \mid x)=F(y-\mu(1)) \mathbb{P}(T=1 \mid X=x)+F(y-\mu(0)) \mathbb{P}(T=0 \mid X=x)$.

Suppose that $\mu(0)<\mu(1)$, that $F$ is absolutely continuous with density function $f$ and that its hazard rate $f(u) /(1-F(u))$ (resp. $f(u) / F(u))$ goes to $+\infty$ as $u \uparrow+\infty$ (resp. $u \downarrow-\infty)$. Then Assumption 3 holds with $G(y)=F(y-\mu(1))$ and $H(y)=F(y-\mu(0))$.

Proof. Let us show that Assumption 3(ii) holds. Let $\varphi(u) \equiv-\ln (1-F(u))$ and note that $\varphi^{\prime}(u)=f(u) /(1-F(u))$. Then

$$
\begin{aligned}
\frac{1-F(y-\mu(0))}{1-F(y-\mu(1))} & =\exp (\varphi(y-\mu(1))-\varphi(y-\mu(0))) \\
& =\exp \left(-\varphi^{\prime}\left(y^{*}\right)(\mu(1)-\mu(0))\right)
\end{aligned}
$$

for some $y^{*}$ between $y-\mu(1)$ and $y-\mu(0)$. Since $\mu(1)>\mu(0)$ and the hazard rate increases without bound as $y \uparrow+\infty$, the expression on the right-hand side tends to zero as $y$ increases. Assumption 3(i) can be verified in the same way.

It is important to note that, aside from regularity conditions, we do not impose any shape restrictions on the mixture components outside of the tails.

We now show that, combined, our exclusion restriction and tail-dominance assumption identify all elements of the mixture model.

THEOREM 1 (Identification). Under Assumptions $1-3, G, H$, and $\lambda$ are identified.

Proof. The proof is constructive. Fix $x^{\prime} \in \mathcal{X}$ and choose $x^{\prime \prime} \in \mathcal{X}$ so that $\lambda\left(x^{\prime}\right) \neq$ $\lambda\left(x^{\prime \prime}\right)$. Then re-arranging (1.1) gives

$$
\begin{aligned}
\frac{F\left(y \mid x^{\prime}\right)}{F\left(y \mid x^{\prime \prime}\right)} & =\frac{1+\lambda\left(x^{\prime}\right)(G(y) / H(y)-1)}{1+\lambda\left(x^{\prime \prime}\right)(G(y) / H(y)-1)}, \\
\frac{1-F\left(y \mid x^{\prime}\right)}{1-F\left(y \mid x^{\prime \prime}\right)} & =\frac{\lambda\left(x^{\prime}\right)+((1-H(y)) /(1-G(y)))\left(1-\lambda\left(x^{\prime}\right)\right)}{\lambda\left(x^{\prime \prime}\right)+((1-H(y)) /(1-G(y)))\left(1-\lambda\left(x^{\prime \prime}\right)\right)} .
\end{aligned}
$$

Taking limits, Assumption 3 further implies that

$$
\begin{aligned}
\zeta^{-}\left(x^{\prime}, x^{\prime \prime}\right) & \equiv \lim _{y \downarrow-\infty} \frac{F\left(y \mid x^{\prime}\right)}{F\left(y \mid x^{\prime \prime}\right)}=\frac{1-\lambda\left(x^{\prime}\right)}{1-\lambda\left(x^{\prime \prime}\right)}, \\
\zeta^{+}\left(x^{\prime}, x^{\prime \prime}\right) & \equiv \lim _{y \uparrow+\infty} \frac{1-F\left(y \mid x^{\prime}\right)}{1-F\left(y \mid x^{\prime \prime}\right)}=\frac{\lambda\left(x^{\prime}\right)}{\lambda\left(x^{\prime \prime}\right)} .
\end{aligned}
$$

These two equations can be solved for the mixing proportion at $x^{\prime}$, yielding

$$
\lambda\left(x^{\prime}\right)=\frac{1-\zeta^{-}\left(x^{\prime \prime}, x^{\prime}\right)}{\zeta^{+}\left(x^{\prime \prime}, x^{\prime}\right)-\zeta^{-}\left(x^{\prime \prime}, x^{\prime}\right)} .
$$


Since $\lambda$ is nonconstant, for any $x^{\prime} \in \mathcal{X}$, there exists a $x^{\prime \prime} \in \mathcal{X}$ for which such a system of equations can be constructed. The function $\lambda$ is therefore identified on its entire support. To establish identification of $G$ and $H$, first note that

$G(y)-H(y)=\frac{F\left(y \mid x^{\prime \prime}\right)-F\left(y \mid x^{\prime}\right)}{\lambda\left(x^{\prime \prime}\right)-\lambda\left(x^{\prime}\right)}$

follows from (1.1). Then, evaluating (1.1) in $x^{\prime \prime}$ and re-arranging the resulting expression for $F\left(y \mid x^{\prime \prime}\right)$ gives

$$
\begin{aligned}
H(y) & =F\left(y \mid x^{\prime \prime}\right)-(G(y)-H(y)) \lambda\left(x^{\prime \prime}\right) \\
& =F\left(y \mid x^{\prime \prime}\right)-\frac{\lambda\left(x^{\prime \prime}\right)}{\lambda\left(x^{\prime \prime}\right)-\lambda\left(x^{\prime}\right)}\left(F\left(y \mid x^{\prime \prime}\right)-F\left(y \mid x^{\prime}\right)\right),
\end{aligned}
$$

which is identified. Furthermore, using (1.2) we can write

$H(y)=F\left(y \mid x^{\prime \prime}\right)-\frac{1}{1-\zeta^{+}\left(x^{\prime}, x^{\prime \prime}\right)}\left(F\left(y \mid x^{\prime \prime}\right)-F\left(y \mid x^{\prime}\right)\right)$.

Plugging this expression for $H(y)$ back into the mixture representation of $F\left(y \mid x^{\prime \prime}\right)$ as in (1.1) further yields

$G(y)=F\left(y \mid x^{\prime \prime}\right)-\frac{1}{1-\zeta^{-}\left(x^{\prime}, x^{\prime \prime}\right)}\left(F\left(y \mid x^{\prime \prime}\right)-F\left(y \mid x^{\prime}\right)\right)$,

again using (1.2). This shows that both component distributions are identified, concluding the proof.

If we only assume one-sided tail dominance, then either $G$ or $H$ remains identified.

COROLLARY 1 (One-sided tail dominance). Under Assumptions 1 and 2, G is identified if Assumption 3(i) holds and $\mathrm{H}$ is identified if Assumption 3(ii) holds.

Proof. We consider identification of $H$. Let $x^{\prime}, x^{\prime \prime}$ be as in the proof of Theorem 1. Under Assumption 3(ii), we can still determine $\zeta^{+}\left(x^{\prime}, x^{\prime \prime}\right)=$ $\lambda\left(x^{\prime}\right) / \lambda\left(x^{\prime \prime}\right)$, from which we can learn the ratio $1 /\left(1-\zeta^{+}\left(x^{\prime}, x^{\prime \prime}\right)\right)$. Together with (1.5), this yields $H$. This concludes the proof of the corollary.

The following example illustrates the usefulness of Corollary 1.

Example 5 (Stochastic volatility)

Consider a two-regime stochastic volatility model, which is a special case of Example 2. Assume that the outcome variable $Y$ has mean zero and conditional variance

$T \sigma_{G}^{2}+(1-T) \sigma_{H}^{2}$

for positive constants $\sigma_{G}^{2}$ and $\sigma_{H}^{2}$. Suppose that $\sigma_{G}^{2}>\sigma_{H}^{2}$. Then $G$ is the distribution associated with a regime that is characterized by relatively higher volatility. In this case, both tails of $G$ dominate those of $H$. Hence, in Assumption 3, 
Condition (ii) holds but Condition (i) fails. Nevertheless, the distribution $H$ of the lower-volatility regime remains identified.

Our identification result suggests plug-in estimators of the mixing proportions and the component distributions.

The proof of Theorem and Equations (1.5)-(1.6) in particular, further show that our mixture model yields overidentifying restrictions as soon as the instrument can take on more than two values. We turn to estimation in the Section 2, where we also construct a statistic for a specification test that exploits the invariance of the formulae for $G$ and $H$ in Equations (1.5)-(1.6) to the values $x^{\prime}, x^{\prime \prime} .^{3}$

\section{ESTIMATION}

To motivate the construction of our estimators, we first note that the structure of the model in (1.1) continues to hold when we aggregate across $x$. Extending our notation to

$$
F(y \mid A) \equiv \mathbb{P}(Y \leq y \mid X \in A), \quad \lambda(A) \equiv \sum_{x \in A} \lambda(x) \mathbb{P}(X=x \mid X \in A),
$$

for any $A \subset \mathcal{X}$, we have

$$
F(y \mid A)=G(y) \lambda(A)+H(y)(1-\lambda(A)),
$$

which is of the same form as (1.1). Furthermore, the proof of Theorem 1 continues to go through for (2.1); replacing $x^{\prime}$ with $A$ and $x^{\prime \prime}$ with $\mathcal{X}-A$ does not alter the argument.

We will assume from now on that $X$ is discrete. As will become apparent, this only entails a loss of generality for the estimation of the function $\lambda$, as our estimator will only yield a discretized approximation to it. Extending our results to continuous $X$ would complicate the exposition greatly and we feel that it would only distract from our main argument.

We will work under the following sampling condition.

Assumption 4. $\left(Y_{1}, X_{1}\right), \ldots,\left(Y_{n}, X_{n}\right)$ is a random sample on $(Y, X)$.

For each $A \subset \mathcal{X}$, let

$$
F_{n}(y \mid A) \equiv n_{A}^{-1} \sum_{i=1}^{n} 1\left\{Y_{i} \leq y, X_{i} \in A\right\}
$$

where $n_{A} \equiv \sum_{i=1}^{n} 1\left\{X_{i} \in A\right\}$.

For each pair of disjoint subsets $A, B$ of $\mathcal{X}$, we can generalize (1.2) to

$$
\begin{aligned}
\zeta^{-}(A, B) & \equiv \lim _{y \downarrow-\infty} \frac{F(y \mid A)}{F(y \mid B)}=\frac{1-\lambda(A)}{1-\lambda(B)}, \\
\zeta^{+}(A, B) & \equiv \lim _{y \uparrow+\infty} \frac{1-F(y \mid A)}{1-F(y \mid B)}=\frac{\lambda(A)}{\lambda(B)} .
\end{aligned}
$$


For any subsample of size $m$ and integers $l_{m}$ and $\kappa_{m}$, let $\ell_{m}$ and $r_{m}$ denote the $\left(l_{m}+1\right)$ th and $\left(m-\kappa_{m}\right)$ th order statistics of $Y$ in this subsample. We estimate the quantities in (2.2) by

$\zeta_{n}^{-}(A, B) \equiv \frac{F_{n}\left(\ell_{n_{B}} \mid A\right)}{F_{n}\left(\ell_{n_{B}} \mid B\right)}, \quad \zeta_{n}^{+}(A, B) \equiv \frac{1-F_{n}\left(r_{n_{B}} \mid A\right)}{1-F_{n}\left(r_{n_{B}} \mid B\right)}$,

respectively. In our asymptotic theory, we will choose $l_{n_{B}}$ and $\kappa_{n_{B}}$ so that $\ell_{n_{B}} \downarrow$ $-\infty$ and $r_{n_{B}} \uparrow+\infty$ as $n \uparrow+\infty$, at an appropriate rate.

Estimators of both the mixing proportions and the component distributions follow readily along the lines of the proof of Theorem 1; see below. Since their asymptotic distribution will be driven by the large-sample behavior of the estimators of the quantities in (2.3), we start by deriving the statistical properties of these estimators.

\subsection{Asymptotic theory for intermediate quantiles}

Throughout this section, we fix disjoint sets $A, B$ and consider the asymptotic behavior of the estimators in (2.3).

Consistency only requires the following rate conditions.

Assumption 5 (Order statistics). $\iota_{n_{B}} / \sqrt{n_{B} \ln \ln n_{B}} \uparrow+\infty \quad$ and $\kappa_{n_{B}} / \sqrt{n_{B} \ln \ln n_{B}} \uparrow+\infty$ as $n \uparrow+\infty$.

THEOREM 2 (Consistency). If Assumptions 1-5 hold,

$\zeta_{n}^{-}(A, B) \stackrel{p}{\rightarrow} \zeta^{-}(A, B), \quad \zeta_{n}^{+}(A, B) \stackrel{p}{\rightarrow} \zeta^{+}(A, B)$,

as $n \uparrow+\infty$.

Proof. We prove the theorem for $\zeta_{n}^{+}$; the proof for $\zeta_{n}^{-}$follows in a similar fashion. Write

$\zeta_{n}^{+}-\zeta^{+}=\left(\zeta_{n}^{+}-\zeta^{\kappa_{n}}\right)+\left(\zeta^{\kappa_{n}}-\zeta^{+}\right)$,

for $\zeta^{\kappa_{n}} \equiv\left(1-F\left(r_{n_{B}} \mid A\right)\right) /\left(1-F\left(r_{n_{B}} \mid B\right)\right)$. For the second right-hand side term in (2.4) we have

$$
\begin{aligned}
\zeta^{\kappa_{n_{B}}-\zeta^{+}} & =\left(\frac{\lambda(A)+\frac{1-H\left(r_{n_{B}}\right)}{1-G\left(r_{n_{B}}\right)}(1-\lambda(A))}{\lambda(B)+\frac{1-H\left(r_{n_{B}}\right)}{1-G\left(r_{n_{B}}\right)}(1-\lambda(B))}-\frac{\lambda(A)}{\lambda(B)}\right) \\
& =O_{p}\left(\frac{1-H\left(r_{n_{B}}\right)}{1-G\left(r_{n_{B}}\right)}\right)=o_{p}(1),
\end{aligned}
$$

by Assumptions 3(ii) and 5. To deal with the first right-hand side term in (2.4), recall that

$\zeta_{n}^{+}-\zeta^{\kappa_{n}}=\frac{1-F_{n}\left(r_{n_{B}} \mid A\right)}{1-F_{n}\left(r_{n_{B}} \mid B\right)}-\frac{1-F\left(r_{n_{B}} \mid A\right)}{1-F\left(r_{n_{B}} \mid B\right)}$. 
Letting $\mathbb{G}_{n}(y \mid S) \equiv \sqrt{n_{S}}\left(F_{n}(y \mid S)-F(y \mid S)\right)$ for any $S \subset \mathcal{X}$ we thus have that

$$
\begin{aligned}
\zeta_{n}^{+}-\zeta^{\kappa_{n}} & =\frac{\left(1-F\left(r_{n_{B}} \mid A\right)\right) \mathbb{G}_{n}\left(r_{n_{B}} \mid B\right) / \sqrt{n_{B}}-\left(1-F\left(r_{n_{B}} \mid B\right)\right) \mathbb{G}_{n}\left(r_{n_{B}} \mid A\right) / \sqrt{n_{A}}}{\left(1-F_{n}\left(r_{n_{B}} \mid B\right)\right)\left(1-F\left(r_{n_{B}} \mid B\right)\right)} \\
& =\frac{\sqrt{n_{B}}}{\kappa_{n_{B}}}\left(\zeta^{\kappa_{n}} \mathbb{G}_{n}\left(r_{n_{B}} \mid B\right)-\sqrt{\frac{n_{B}}{n_{A}}} \mathbb{G}_{n}\left(r_{n_{B}} \mid A\right)\right) \\
& =O_{\text {a.s. }}\left(\frac{\sqrt{n_{B} \ln \ln n_{B}}}{\kappa_{n_{B}}}\right),
\end{aligned}
$$

where the second equality uses $1-F_{n}\left(r_{n_{B}} \mid B\right)=\kappa_{n_{B}} / n_{B}$ and the last one follows by the law of the iterated logarithm for empirical processes. Thus, from Assumption 5 it follows that $\left|\zeta_{n}^{+}-\zeta^{\kappa_{n}}\right|=o_{p}(1)$. This completes the proof.

Deriving the limit distribution requires some more care, and three more assumptions. We first impose the following regularity condition on the component distributions.

Assumption 6. $G$ and $H$ are absolutely continuous on $\mathbb{R}$.

This assumption is very weak. Note that, as we do not require the existence of moments of the component distributions, our results also apply to heavy-tailed distributions such as Cauchy and Pareto distributions.

We will complement Assumption 5 with an additional rate condition.

Assumption 7 (Order statistics (cont'd.)). $l_{n_{B}} / n_{B} \downarrow 0$ and $\kappa_{n_{B}} / n_{B} \downarrow 0$ as $n \uparrow$ $+\infty$.

Where Assumption 5 required the order statistics to grow to ensure consistency, this assumption bounds this growth rate so that appropriately scaled versions of $\zeta_{n}^{+}$and $\zeta_{n}^{-}$have a limit distribution.

Finally, we will use an additional condition on the relative tails of the component distributions.

Assumption 8 (Tail rates).

(i) $G\left(\ell_{n_{B}}\right) / H\left(\ell_{n_{B}}\right)=o_{p}\left(1 / \sqrt{l_{n_{B}}}\right)$; and

(ii) $\left(1-H\left(r_{n_{B}}\right)\right) /\left(1-G\left(r_{n_{B}}\right)\right)=o_{p}\left(1 / \sqrt{\kappa_{n_{B}}}\right)$.

Assumption 8 rules out distributions whose tails vanish too quickly and ensures that the limit distributions are free of asymptotic bias. We comment on Assumption 8 after we derive the limit distributions of our estimators.

Let $\rho_{A, B} \equiv \mathbb{P}(X \in B) / \mathbb{P}(X \in A)$. Note that $0<\rho_{A, B}<+\infty$ because of random sampling. Introduce

$\sigma_{-}^{2}(A, B) \equiv \zeta^{-}(A, B)^{2}+\rho_{A, B} \zeta^{-}(A, B)$,

$\sigma_{+}^{2}(A, B) \equiv \zeta^{+}(A, B)^{2}+\rho_{A, B} \zeta^{+}(A, B)$. 
Theorem 2 provides the asymptotic properties of the estimators in (2.2) and is the main building block for our subsequent results.

THEOREM 3 (Asymptotic normality). If Assumptions 1-8 hold then, as $n \uparrow$ $+\infty$

$$
\begin{aligned}
& \sqrt{l_{n_{B}}}\left(\zeta_{n}^{-}(A, B)-\zeta^{-}(A, B)\right) \stackrel{d}{\rightarrow} \mathcal{N}\left(0, \sigma_{-}^{2}(A, B)\right) \\
& \sqrt{\kappa_{n_{B}}}\left(\zeta_{n}^{+}(A, B)-\zeta^{+}(A, B)\right) \stackrel{d}{\rightarrow} \mathcal{N}\left(0, \sigma_{+}^{2}(A, B)\right)
\end{aligned}
$$

and these two estimators are asymptotically independent.

Proof. We focus on the limit behavior of $\sqrt{\kappa_{n_{B}}}\left(\zeta_{n}^{+}-\zeta^{+}\right)$here; the proof of the result for $\sqrt{l_{n}}\left(\zeta_{n}^{-}-\zeta^{-}\right)$follows along similar lines.

As in the proof of Theorem 2, write

$$
\sqrt{\kappa_{n_{B}}}\left(\zeta_{n}^{+}-\zeta^{+}\right)=\sqrt{\kappa_{n_{B}}}\left(\zeta_{n}^{+}-\zeta^{\kappa_{n_{B}}}\right)+\sqrt{\kappa_{n_{B}}}\left(\zeta^{\kappa_{n_{B}}}-\zeta^{+}\right)
$$

$$
\text { for } \zeta^{\kappa_{n_{B}}} \equiv\left(1-F\left(r_{n_{B}} \mid A\right)\right) /\left(1-F\left(r_{n_{B}} \mid B\right)\right) \text {. Assumption } 8 \text { implies that }
$$

$$
\sqrt{\kappa_{n_{B}}}\left(\zeta^{\kappa_{n_{B}}}-\zeta^{+}\right)=\sqrt{\kappa_{n_{B}}} O_{p}\left(\frac{1-H\left(r_{n_{B}}\right)}{1-G\left(r_{n_{B}}\right)}\right)=o_{p}(1)
$$

Hence, the second right-hand side term in (2.5) is asymptotically negligible.

We now turn to the first term in (2.5). From the proof of Theorem 2, we have that

$$
\sqrt{\kappa_{n_{B}}}\left(\zeta_{n}^{+}-\zeta^{\kappa_{n}}\right)=\sqrt{\frac{n_{B}}{\kappa_{n_{B}}}}\left(\zeta^{\kappa_{n}} \mathbb{G}_{n}\left(r_{n_{B}} \mid B\right)-\sqrt{\frac{n_{B}}{n_{A}}} \mathbb{G}_{n}\left(r_{n_{B}} \mid A\right)\right),
$$

where $\mathbb{G}_{n}(y \mid S) \equiv \sqrt{n_{S}}\left(F_{n}(y \mid S)-F(y \mid S)\right)$ for any $S \subset \mathcal{X}$. Let $\alpha_{n}(u) \equiv$ $\sqrt{n}\left(\mathcal{U}_{n}(u)-u\right)$ for $\mathcal{U}_{n}$ the empirical cumulative distribution of an i.i.d. sample of size $n$ from a uniform distribution on $[0,1]$. By Assumption 6, $F(y \mid S)$ is continuous in $y$ for all $S \subset \mathcal{X}$. Therefore,

$$
\mathbb{G}_{n}(y \mid A)=\alpha_{n_{A}}(1-F(y \mid A)) \text { and } \mathbb{G}_{n}(y \mid B)=\alpha_{n_{B}}(1-F(y \mid B))
$$

by an application of the probability integral transform. Hence, we may write

$$
\begin{aligned}
\sqrt{\kappa_{n_{B}}}\left(\zeta_{n}^{+}-\zeta^{\left.\kappa_{n_{B}}\right)=}\right. & \zeta^{\kappa_{n_{B}}} \sqrt{\frac{n_{B}}{\kappa_{n_{B}}}} \alpha_{n_{B}}\left(1-F\left(r_{n_{B}} \mid B\right)\right) \\
& -\sqrt{\frac{n_{B}}{\kappa_{n_{B}}}} \sqrt{\frac{n_{B}}{n_{A}}} \alpha_{n_{A}}\left(1-F\left(r_{n_{B}} \mid A\right)\right) .
\end{aligned}
$$

We study the asymptotic behavior of each of the right-hand side terms in turn. 
Start with the first right-hand side term in (2.6). From the definition of the order statistic $r_{n_{B}}$ we find, by adding and subtracting $F_{n}\left(r_{n_{B}} \mid B\right)$, that

$1-F\left(r_{n_{B}} \mid B\right)=\frac{\kappa_{n_{B}}}{n_{B}}\left(1+\frac{\sqrt{n_{B}}}{\kappa_{n_{B}}} \mathbb{G}_{n}\left(r_{n_{B}} \mid B\right)\right) ;$

or, defining $\varepsilon_{n} \equiv-\sqrt{n_{B}} / \kappa_{n_{B}} \mathbb{G}_{n}\left(r_{n_{B}} \mid B\right)$,

$1-F\left(r_{n_{B}} \mid B\right)=\frac{\kappa_{n_{B}}}{n_{B}}\left(1-\varepsilon_{n}\right)$.

Therefore, we can write

$\zeta^{\kappa_{n}} \sqrt{\frac{n_{B}}{\kappa_{n_{B}}}} \alpha_{n_{B}}\left(1-F\left(r_{n_{B}} \mid B\right)\right)=\sqrt{2} \zeta^{\kappa_{n}} \sqrt{\frac{n_{B}}{2 \kappa_{n_{B}}}} \alpha_{n_{B}}\left(\frac{2 \kappa_{n_{B}}}{n_{B}} \frac{1-\varepsilon_{n}}{2}\right)$.

By the law of the iterated logarithm together with Assumption 5,

$\varepsilon_{n}=-\frac{\sqrt{n_{B}}}{\kappa_{n_{B}}} O_{a . s .}\left(\sqrt{\ln \ln n_{B}}\right)=O_{\text {a.s. }}\left(\frac{\sqrt{n_{B} \ln \ln n_{B}}}{\kappa_{n_{B}}}\right)=o_{a . s .}(1)$.

Hence $\left(1-\varepsilon_{n}\right) / 2$ converges almost surely to $1 / 2$; and $\left(1-\varepsilon_{n}\right) / 2 \in(0,1)$ for $n$ large enough. We may then apply Theorem 2.1 in Einmahl (1992) to establish the convergence in distribution of $\sqrt{\frac{n_{B}}{2 \kappa_{n}}} \alpha_{n_{B}}\left(\frac{2 \kappa_{n_{B}}}{n_{B}} \frac{1-\varepsilon_{n}}{2}\right)$ to a normal random variable with mean zero and variance $1 / 2$. This, together with Equation (2.7) and an application of Slutsky's theorem, implies that

$\sqrt{\frac{n_{B}}{\kappa_{n_{B}}}} \zeta^{\kappa_{n_{B}}} \alpha_{n_{B}}\left(1-F\left(r_{n_{B}} \mid B\right)\right) \stackrel{d}{\rightarrow} \zeta^{+} Z_{B}^{+}$

where $Z_{B}^{+}$is a standard normal random variable.

Now turn to the second right-hand side term in (2.6). First observe that

$$
1-F\left(r_{n_{B}} \mid A\right)=\zeta^{\kappa_{n}}\left(1-F\left(r_{n_{B}} \mid B\right)\right)=\zeta^{\kappa_{n}} \frac{\kappa_{n_{B}}}{n_{B}}\left(1-\varepsilon_{n}\right)
$$

Using $\rho_{A, B}=\lim _{n \uparrow+\infty} n_{B} / n_{A}$, this gives

$\sqrt{\frac{n_{B}}{\kappa_{n_{B}}}} \sqrt{\frac{n_{B}}{n_{A}}} \alpha_{n_{A}}\left(1-F\left(r_{n_{B}} \mid A\right)\right)=\sqrt{2 \rho_{A}, B \zeta^{+}} \sqrt{\frac{n_{A}}{2 \tilde{\kappa}_{n_{A}}}} \alpha_{n_{A}}\left(\frac{2 \tilde{\kappa}_{n_{A}}}{n_{A}} \frac{1-\varepsilon_{n}}{2}\right)+o_{p}(1)$,

where $\tilde{\kappa}_{n_{A}} \equiv\left(\kappa_{n_{B}} \zeta^{\kappa_{n_{B}}}\right) /\left(n_{B} / n_{A}\right)$. As $\tilde{\kappa}_{n_{A}}$ satisfies Assumption (1.5) of Theorem 2.1 in Einmahl (1992), we may apply his theorem again to obtain

$\sqrt{\frac{n_{B}}{\kappa_{n_{B}}}} \sqrt{\frac{n_{B}}{n_{A}}} \alpha_{n_{A}}\left(1-F\left(r_{n_{B}} \mid A\right)\right) \stackrel{d}{\rightarrow} \sqrt{\rho_{A, B} \zeta^{+}} Z_{A}^{+}$, 
where $Z_{A}^{+}$is a standard-normal random variable which, because of random sampling, is independent of $Z_{B}^{+}$.

Combining (2.6) with (2.8) and (2.9) then gives

$\sqrt{\kappa_{n_{B}}}\left(\zeta_{n}^{+}-\zeta^{\kappa_{n} B}\right) \stackrel{d}{\rightarrow} \zeta^{+} Z_{B}^{+}-\sqrt{\rho_{A, B} \zeta^{+}} Z_{A}^{+}$,

as claimed. This concludes the proof.

We finish this section with two examples that specialize Assumption 8 to densities with log-concave tails and Pareto tails, respectively. In both cases, Assumption 8 is implied by the rate conditions in Assumption 7.

Example 6 (Log-concave tails)

Suppose that $G$ and $H$ have log-concave tails; and for notational simplicity, assume that

$-\ln (1-G(y)) \sim\left(\frac{y}{\sigma_{G}^{+}}\right)^{\alpha_{G}^{+}}, \quad-\ln (1-H(y)) \sim\left(\frac{y}{\sigma_{H}^{+}}\right)^{\alpha_{H}^{+}}, \quad$ as $y \uparrow+\infty$

for real numbers $\alpha_{G}^{+}, \alpha_{H}^{+}>1$ and $\sigma_{G}^{+}, \sigma_{H}^{+}>0$, and

$-\ln G(y) \sim\left(\frac{-y}{\sigma_{G}^{-}}\right)^{\alpha_{G}^{-}}, \quad-\ln H(y) \sim\left(\frac{-y}{\sigma_{H}^{-}}\right)^{\alpha_{H}^{-}}, \quad$ as $y \downarrow-\infty$,

for real numbers $\alpha_{G}^{-}, \alpha_{H}^{-}>1$ and $\sigma_{G}^{-}, \sigma_{H}^{-}>0$. Then Assumption 7 implies Assumption 8 if both

(i) $\alpha_{G}^{+}<\alpha_{H}^{+}$, or $\alpha_{G}^{+}=\alpha_{H}^{+}$and $\sigma_{G}^{+}>\sigma_{H}^{+}$; and

(ii) $\alpha_{G}^{-}>\alpha_{H}^{-}$, or $\alpha_{G}^{-}=\alpha_{H}^{-}$and $\sigma_{G}^{-}<\sigma_{H}^{-}$

hold.

Proof. We verify the second rate; the first follows similarly. Throughout, fix the set $B$. Assumptions 3(ii) and 7 imply that

$$
\begin{aligned}
1-F\left(r_{n_{B}} \mid B\right) & =\left(1-G\left(r_{n_{B}}\right)\right) \lambda(B)+\left(1-H\left(r_{n_{B}}\right)\right)(1-\lambda(B)) \\
& =\left(1-G\left(r_{n_{B}}\right)\right)\left(\lambda(B)+o_{p}(1)\right) .
\end{aligned}
$$

Further, because $\kappa_{n_{B}} / n_{B}=1-F_{n}\left(r_{n_{B}} \mid B\right)$, adding and subtracting $F\left(r_{n_{B}} \mid B\right)$ gives

$$
\begin{aligned}
\frac{\kappa_{n_{B}}}{n_{B}} & =\left(1-F\left(r_{n_{B}} \mid B\right)\right)+\left(F_{n}\left(r_{n_{B}} \mid B\right)-F\left(r_{n_{B}} \mid B\right)\right) \\
& =\left(1-F\left(r_{n_{B}} \mid B\right)\right)+O_{\text {a.s. }}\left(\sqrt{\left(\ln \ln n_{B}\right) / n_{B}}\right) .
\end{aligned}
$$


Because $\left(\ln \ln n_{B}\right) / n_{B} \rightarrow 0$, put together, we find

$\frac{\kappa_{n_{B}}}{n_{B}}=C\left(1-G\left(r_{n_{B}}\right)\right)\left(1+o_{p}(1)\right)$

for some constant $C$. Since $G$ and $H$ have log-concave tails, it follows from this expression that $r_{n_{B}}$ behaves asymptotically like $\sqrt[\alpha_{G}^{+}]{\ln n_{B}}$. And since

$$
\frac{1-H\left(r_{n_{B}}\right)}{1-G\left(r_{n_{B}}\right)} \sim \exp \left\{\left(\frac{r_{n_{B}}}{\sigma_{G}^{+}}\right)^{\alpha_{G}^{+}}-\left(\frac{r_{n_{B}}}{\sigma_{H}^{+}}\right)^{\alpha_{H}^{+}}\right\}
$$

we have that

$$
\frac{1-H\left(r_{n_{B}}\right)}{1-G\left(r_{n_{B}}\right)}=\left\{\begin{array}{ll}
O_{p}\left(\operatorname { e x p } \left(-\left(\ln n_{B}\right)^{\left.\left.\alpha_{H}^{+} / \alpha_{G}^{+}\right)\right)}\right.\right. & \text {if } \alpha_{H}^{+}>\alpha_{G}^{+} \\
O_{p}\left(1 / n_{B}\right) & \text { if } \alpha_{H}^{+}=\alpha_{G}^{+} \text {and } \sigma_{H}^{+}<\sigma_{G}^{+}
\end{array},\right.
$$

from which the conclusion follows.

Example 6 does not cover location models with log-concave distributions in the case when the $\alpha$ and $\sigma$ parameters of $H$ equal those of $G$. This includes the location model with Gaussian errors, for which $\alpha=2$ and $\sigma$ is the common standard deviation. While our estimator remains consistent in such cases, we do not know of general results on tail empirical processes that would yield the asymptotic distribution of the estimator in this knife-edge case. To assess the extent to which the failure of Assumption 8 may play a role for inference, our simulation experiments in Section 3 include a Gaussian location model.

Example 7 (Pareto tails)

Let $C$ denote a generic constant. Suppose that $G$ and $H$ have Pareto tails, i.e.,

$$
(1-G(y)) \sim C y^{-\alpha_{G}^{+}}, \quad(1-H(y)) \sim C y^{-\alpha_{H}^{+}}, \quad \text { as } y \uparrow+\infty,
$$

for positive real numbers $\alpha_{H}^{+}>\alpha_{G}^{+}$and

$G(y) \sim C(-y)^{-\alpha_{G}^{-}}, \quad H(y) \sim C(-y)^{-\alpha_{H}^{-}}, \quad$ as $y \downarrow-\infty$,

for positive real numbers $\alpha_{G}^{-}<\alpha_{H}^{-}$. Then Assumption 7 implies Assumption 8.

Proof. The argument is very similar to the one that was used to verify Example 6. We focus on the right tail; the argument for the left tail is similar. We have $\frac{\kappa_{n_{B}}}{n_{B}}=\left(1-G\left(r_{n_{B}}\right)\right)\left(1+o_{p}(1)\right)=C r^{-\alpha_{G}^{+}}\left(1+o_{p}(1)\right)$.

Assumption 8 requires that $\left(1-H\left(r_{n_{B}}\right)\right) /\left(1-G\left(r_{n_{B}}\right)\right)=o\left(1 / \sqrt{\kappa_{n}}\right)$, that is, that $r_{n_{B}}^{\alpha_{G}^{+}-\alpha_{H}^{+}}=o_{p}\left(1 / \sqrt{\kappa_{n_{B}}}\right)$. This rate condition is satisfied when

$$
\left(\frac{n_{B}}{\kappa_{n_{B}}}\right)^{\frac{\alpha_{G}^{+}-\alpha_{H}^{+}}{\alpha_{G}^{+}}}=o_{p}\left(\frac{1}{\sqrt{\kappa_{n_{B}}}}\right)
$$


which can be achieved by setting $\kappa_{n_{B}}=o\left(n_{B}^{\gamma^{+}}\right)$for

$\gamma^{+} \equiv \frac{\alpha_{H}^{+}-\alpha_{G}^{+}}{\alpha_{H}^{+}-\alpha_{G}^{+} / 2}$.

This condition is weaker than Assumption 7 and is therefore implied by it.

Example 7 shows that our methods are well suited to deal with Pareto tails. Pareto tails show up in many economic applications. A time-honored example is income and wealth distributions (Atkinson, Piketty, and Saez, 2011), which are often modeled as a log-normal for most quantiles, combined with a Pareto right tail. More generally, "power laws" have become a popular tool in finance, in studies of firm growth, and in urban economics (see Gabaix, 2009 for a recent survey, and Acemoglu, Carvalho, Ozdaglar, and Tabaz-Salehi, 2012 for an application to business cycles.) Many recent models of monopolistic competition, as used in international trade for instance, also assume that productivities are Pareto-distributed (Arkolakis, Costinot, and Rodriguez-Clare, 2012).

Let us focus on the right tail condition. Identification only requires that the tail index of $H$ be larger than that of $G$, that is, $\alpha_{H}^{+}>\alpha_{G}^{+}$. Let $c^{+} \equiv \alpha_{H}^{+} / \alpha_{G}^{+}>1$. Equation (2.10) then gives a convergence rate arbitrarily close to $n^{-\beta^{+} / 2}$ for $\beta^{+}=2\left(c^{+}-1\right) /\left(2 c^{+}-1\right)$. For example, if $c^{+}=2$ then $\beta^{+}=2 / 3$ and our estimators will converge slightly slower than $n^{-1 / 3}$. However, as $c^{+}$increases, $\beta^{+}$ becomes closer to one and our estimators will converge at close to the $n^{-1 / 2}$ parametric rate.

\subsection{Mixing proportions}

Fix $x \in \mathcal{X}$ and consider estimating $\lambda(x)$. Set $A=\mathcal{X}-x$ and $B=x$ in (2.2) and solve for $\lambda(x)$ to get

$\lambda(x)=\frac{1-\zeta^{-}(A, x)}{\zeta^{+}(A, x)-\zeta^{-}(A, x)}$.

The mixing proportion $\lambda$ need not be a strictly monotonic function. Estimating $\lambda(x)$ by an average of plug-in estimates of (1.3) could therefore be problematic, as the denominator in (1.3) can be zero or be arbitrarily close to it for some pairs of values $\left(x^{\prime}, x^{\prime \prime}\right)$.

We instead estimate the mixing proportion at $X=x$ by the plug-in estimator

$\lambda_{n}(x) \equiv \frac{1-\zeta_{n}^{-}(A, x)}{\zeta_{n}^{+}(A, x)-\zeta_{n}^{-}(A, x)}$.

This estimator uses observations with $X_{i} \neq x$ in a way that immunizes it against small or zero denominators. 
To present the asymptotic variance of this estimator, we need to define

$$
\begin{aligned}
& d^{-}(x) \equiv \frac{1-\zeta^{+}(A, x)}{\left(\zeta^{+}(A, x)-\zeta^{-}(A, x)\right)^{2}}, \\
& d^{+}(x) \equiv \frac{\zeta^{-}(A, x)-1}{\left(\zeta^{+}(A, x)-\zeta^{-}(A, x)\right)^{2}} .
\end{aligned}
$$

The speed of convergence and the asymptotic distribution of the $\lambda_{n}(x)$ depend on the ratio $c_{x} \equiv \lim _{n \uparrow+\infty} l_{n_{x}} / \kappa_{n_{x}}$.

THEOREM 4 (Mixing proportions). Under the conditions of Theorem 2,

$\left|\lambda_{n}(x)-\lambda(x)\right|=o_{p}(1)$,

as $n \uparrow+\infty$.

Under the conditions of Theorem 3,

$$
\begin{aligned}
& \sqrt{{\overline{n_{x}}}_{x}}\left(\lambda_{n}(x)-\lambda(x)\right) \stackrel{d}{\rightarrow} \mathcal{N}\left(0, d^{-}(x)^{2} \sigma_{-}^{2}(A, x)+c_{x} d^{+}(x)^{2} \sigma_{+}^{2}(A, x)\right) \text { if } c_{x}<+\infty, \\
& \sqrt{\kappa_{n_{x}}}\left(\lambda_{n}(x)-\lambda(x)\right) \stackrel{d}{\rightarrow} \mathcal{N}\left(0, c_{x}^{-1} d^{-}(x)^{2} \sigma_{-}^{2}(A, x)+d^{+}(x)^{2} \sigma_{+}^{2}(A, x)\right) \text { if } c_{x}>0, \\
& \text { as } n \uparrow+\infty .
\end{aligned}
$$

Proof. The consistency claim follows directly from Theorem 2 by an application of the continuous mapping theorem.

To establish the asymptotic distribution, note that Theorem 3 states that

$$
\begin{aligned}
& \sqrt{l_{n_{x}}}\left(\zeta_{n}^{-}(A, x)-\zeta^{-}(A, x)\right) \stackrel{d}{\rightarrow} \mathcal{N}\left(0, \sigma_{-}^{2}(A, x)\right), \\
& \sqrt{\kappa_{n_{x}}}\left(\zeta_{n}^{+}(A, x)-\zeta^{+}(A, x)\right) \stackrel{d}{\rightarrow} \mathcal{N}\left(0, \sigma_{+}^{2}(A, x)\right),
\end{aligned}
$$

and that $\zeta_{n}^{-}(x)$ and $\zeta_{n}^{+}(x)$ are asymptotically independent. An expansion around $\zeta^{-}(A, x)$ and $\zeta^{+}(A, x)$ then yields

$$
\begin{aligned}
\sqrt{l_{n_{x}}}\left(\lambda_{n}(x)-\lambda(x)\right) & =d^{-}(x) \sqrt{l_{n_{x}}}\left(\zeta_{n}^{-}(A, x)-\zeta^{-}(A, x)\right) \\
& +d^{+}(x) \sqrt{\kappa_{n_{x}}}\left(\zeta_{n}^{+}(A, x)-\zeta^{+}(A, x)\right) \sqrt{\frac{l_{n_{x}}}{\kappa_{n_{x}}}}+o_{p}(1),
\end{aligned}
$$

which has the limit distribution stated in the theorem if $c_{x}$ is finite. Also, by the same argument,

$$
\begin{aligned}
\sqrt{\kappa_{n_{x}}}\left(\lambda_{n}(x)-\lambda(x)\right) & =d^{+}(x) \sqrt{\kappa_{n_{x}}}\left(\zeta_{n}^{+}(x)-\zeta^{+}(x)\right) \\
& +d^{-}(x) \sqrt{l_{n_{x}}}\left(\zeta_{n}^{-}(x)-\zeta^{-}(x)\right) \sqrt{\frac{\kappa_{n_{x}}}{l_{n_{x}}}}+o_{p}(1)
\end{aligned}
$$

converges in distribution as stated in the theorem if $c_{x}$ is nonzero. This verifies the claims and proves the theorem. 


\subsection{Component distributions}

To estimate the component distributions, choose $B=\mathcal{X}-A$ so that $A$ and $B$ partition $\mathcal{X}$. Equations (1.5) and (1.6) suggest the estimators

$$
\begin{gathered}
H_{n}(y ; A, B) \equiv F_{n}(y \mid A)-\frac{1}{1-\zeta_{n}^{+}(B, A)}\left(F_{n}(y \mid A)-F_{n}(y \mid B)\right), \\
G_{n}(y ; A, B) \equiv F_{n}(y \mid A)-\frac{1}{1-\zeta_{n}^{-}(B, A)}\left(F_{n}(y \mid A)-F_{n}(y \mid B)\right) .
\end{gathered}
$$

For notational simplicity we now drop $A$ and $B$ from the arguments: $G_{n}(y) \equiv$ $G_{n}(y ; A, B)$ and $H_{n}(y) \equiv H_{n}(y ; A, B)$.

To state their asymptotic behavior, let

$$
\begin{aligned}
& d_{G}(A, B ; y) \equiv \frac{F(y \mid A)-F(y \mid B)}{\left(1-\zeta^{-}(B, A)\right)^{2}}, \\
& d_{H}(A, B ; y) \equiv \frac{F(y \mid A)-F(y \mid B)}{\left(1-\zeta^{+}(B, A)\right)^{2}},
\end{aligned}
$$

and let $\|\cdot\|_{\infty}$ denote the supremum norm.

THEOREM 5. Under the conditions of Theorem 2,

$\left\|G_{n}-G\right\|_{\infty}=o_{p}(1), \quad\left\|H_{n}-H\right\|_{\infty}=o_{p}(1)$,

as $n \uparrow+\infty$.

Under the conditions of Theorem 3,

$$
\begin{aligned}
& \sqrt{r_{n_{A}}}\left(G_{n}(y)-G(y)\right) \stackrel{d}{\rightarrow} \mathcal{N}\left(0, d_{G}(A, B ; y)^{2} \sigma_{-}^{2}(B, A)\right), \\
& \sqrt{\kappa_{n_{A}}}\left(H_{n}(y)-H(y)\right) \stackrel{d}{\rightarrow} \mathcal{N}\left(0, d_{H}(A, B ; y)^{2} \sigma_{+}^{2}(B, A)\right), \\
& \text { as } n \uparrow+\infty, \text { for each } y \in \mathbb{R} .
\end{aligned}
$$

Proof. Consistency follows by Theorem 2 and the Glivenko-Cantelli theorem.

We establish the asymptotic distribution of $G_{n}$; the result for $H_{n}$ follows by the same argument.

First note that

$$
\begin{aligned}
& \sqrt{l_{n_{A}}}\left(G_{n}(y)-G(y)\right)=T_{1}+T_{2}+T_{3} \\
& \text { for } \\
& T_{1} \equiv \sqrt{l_{n_{A}}}\left(F_{n}(y \mid A)-F(y \mid A)\right), \\
& T_{2} \equiv-\frac{1}{1-\zeta^{-}(B, A)} \sqrt{l_{n_{A}}}\left(\left\{F_{n}(y \mid A)-F(y \mid A)\right\}-\left\{F_{n}(y \mid B)-F(y \mid B)\right\}\right), \\
& T_{3} \equiv-\left(F_{n}(y \mid A)-F_{n}(y \mid B)\right) \sqrt{l_{n_{A}}}\left(\frac{1}{1-\zeta_{n}^{-}(B, A)}-\frac{1}{1-\zeta^{-}(B, A)}\right) .
\end{aligned}
$$


By the Glivenko-Cantelli theorem, $T_{1}=o_{p}(1)$ and $T_{2}=o_{p}(1)$ while

$$
T_{3}=-(F(y \mid A)-F(y \mid B)) \sqrt{l_{n_{A}}}\left(\frac{1}{1-\zeta_{n}^{-}(B, A)}-\frac{1}{1-\zeta^{-}(B, A)}\right)+o_{p}(1) .
$$

A linearization of this expression in $\zeta_{n}^{-}(B, A)-\zeta^{-}(B, A)$ together with an application of Theorem 3 to the partition $A, B$ then yields the result.

When $X$ can take on more than two, values there are multiple ways of choosing the sets $A$ and $B$. Inspection of the asymptotic variance does not give clear guidance on how to choose $A$ and $B$ in an optimal manner. An ad-hoc way to proceed when the number of possible choices for $A, B$ is small, is to simply compute estimators for all possible choices. Alternatively, it would be possible to combine estimates based on multiple choices through a minimum-distance procedure. We leave a detailed analysis for future research.

\subsection{Specification testing}

An implication of our model restrictions is that the estimators of $G$ and $H$ in (2.12), when based on different subsets of $\mathcal{X}$, should coincide with one another, up to sampling error. This observation suggests the possibility to test the specification when $X$ can take on more than two values.

Theorem 6 provides the relevant asymptotic distributional result to perform this test. In it we use

$$
\begin{aligned}
\Sigma_{G} & \equiv d_{G}(A, C)\left\{d_{G}(A, C) \sigma_{-}^{2}(C, A)-d_{G}(A, B) \zeta^{-}(C, A) \zeta^{-}(B, A)\right\} \\
& +d_{G}(A, B)\left\{d_{G}(A, B) \sigma_{-}^{2}(B, A)-d_{G}(A, C) \zeta^{-}(C, A) \zeta^{-}(B, A)\right\}
\end{aligned}
$$

and

$$
\begin{aligned}
\Sigma_{H} & \equiv d_{H}(A, C)\left\{d_{H}(A, C) \sigma_{+}^{2}(C, A)-d_{H}(A, B) \zeta^{+}(C, A) \zeta^{+}(B, A)\right\} \\
& +d_{H}(A, B)\left\{d_{H}(A, B) \sigma_{+}^{2}(B, A)-d_{H}(A, C) \zeta^{+}(C, A) \zeta^{+}(B, A)\right\},
\end{aligned}
$$

where the triple $A, B, C$ constitutes any partition of $\mathcal{X}$ and, for any $A$ and $B$, we write

$$
d_{G}(A, B) \equiv \mathbb{E}\left[W(Y) d_{G}(A, B ; Y)\right], \quad d_{H}(A, B) \equiv \mathbb{E}\left[W(Y) d_{H}(A, B ; Y)\right]
$$

for a chosen weight function $W$ that is bounded on $\mathbb{R}$. The choice of these weights should reflect the analyst's concerns about potential violations of our assumptions in the application under study.

THEOREM 6 (Specification testing). Under the conditions of Theorem 3

$$
\lim _{n \uparrow+\infty} \mathbb{P}\left\{\left|\frac{n^{-1} \sum_{i=1}^{n} W\left(Y_{i}\right) G_{n}\left(Y_{i} ; A, B\right)-n^{-1} \sum_{i=1}^{n} W\left(Y_{i}\right) G_{n}\left(Y_{i} ; A, C\right)}{\sqrt{\Sigma_{G}} / \sqrt{l_{n_{A}}}}\right|>z(\tau / 2)\right\}=\tau,
$$


and

$\lim _{n \uparrow+\infty} \mathbb{P}\left\{\left|\frac{n^{-1} \sum_{i=1}^{n} W\left(Y_{i}\right) H_{n}\left(Y_{i} ; A, B\right)-n^{-1} \sum_{i=1}^{n} W\left(Y_{i}\right) H_{n}\left(Y_{i} ; A, C\right)}{\sqrt{\Sigma_{H}} / \sqrt{\kappa_{n_{A}}}}\right|>z(\tau / 2)\right\}=\tau$,

where $z(\tau)$ is the $1-\tau$ quantile of the standard-normal distribution.

Proof. We consider only the case of $G$. The difference $G_{n}(y ; A, B)-$ $G_{n}(y ; A, C)$ equals

$\frac{1}{1-\zeta_{n}^{-}(C, A)}\left(F_{n}(y \mid A)-F_{n}(y \mid C)\right)-\frac{1}{1-\zeta_{n}^{-}(B, A)}\left(F_{n}(y \mid A)-F_{n}(y \mid B)\right)$

for any $y$. An expansion around $\zeta^{-}(C, A)$ and $\zeta^{-}(B, A)$, then shows that the scaled difference $\sqrt{l_{n_{A}}} G_{n}(y ; A, B)-G_{n}(y ; A, C)$ is asymptotically equivalent to $d_{G}(A, C ; y) \sqrt{l_{n_{A}}}\left(\zeta_{n}^{-}(C, A)-\zeta^{-}(C, A)\right)-d_{G}(A, B ; y) \sqrt{l_{n_{A}}}\left(\zeta_{n}^{-}(B, A)-\zeta^{-}(B, A)\right)$.

This holds for any $y$ and, therefore, also for the weighted average over $y$. Together with Theorem 3 , this result then readily yields the asymptotic distribution of the difference $n^{-1} \sum_{i=1}^{n} W\left(Y_{i}\right) G_{n}\left(Y_{i} ; A, B\right)-n^{-1} \sum_{i=1}^{n} W\left(Y_{i}\right) G_{n}\left(Y_{i} ; A, C\right)$ and implies the claim of the theorem.

We leave a detailed analysis of the power properties of this specification test for future research. Here, we provide a consistency result against failure of Assumption 3.

Example 8 (Consistency of the test)

Suppose that $H$ dominates $G$ in both tails. Then $H$ is no longer identified and

$\lim _{n \uparrow+\infty} \mathbb{P}\left\{\left|\frac{n^{-1} \sum_{i=1}^{n} W\left(Y_{i}\right) H_{n}\left(Y_{i} ; A, B\right)-n^{-1} \sum_{i=1}^{n} W\left(Y_{i}\right) H_{n}\left(Y_{i} ; A, C\right)}{\sqrt{\Sigma_{H}} / \sqrt{\kappa_{n_{A}}}}\right|>z\right\}=1$

for any $z$.

Proof. When $H$ dominates $G$ in both tails, a small calculation reveals that

$\zeta_{n}^{+}(A, B)=\zeta^{-}(A, B)+o_{p}(1)$,

and so $\sqrt{\kappa_{n_{A}}}\left|\left(\zeta_{n}^{+}(A, B)-\zeta^{+}(A, B)\right)\right|$ grows without bound as $n \uparrow+\infty$. The conclusion then readily follows from the linearization in the proof of Theorem 6.

\section{SIMULATION EXPERIMENTS}

In our numerical illustrations, we will work with the family of skew-normal distributions (Azzalini, 1985). The skew-normal distribution with location $\mu$, positive 
scale $\sigma$, and skewness parameter $\beta$ multiplies the density of $\mathcal{N}\left(\mu, \sigma^{2}\right)$ by a term that skews it to the right if $\beta>0$ and to the left if $\beta<0$ :

$f(x ; \mu, \sigma, \beta) \equiv \frac{1}{\sigma} \phi\left(\frac{x-\mu}{\sigma}\right) \times \frac{\Phi\left(\beta \frac{x-\mu}{\sigma}\right)}{\Phi(0)}$.

Its mean and variance are $\mu+\sigma \delta \sqrt{\frac{2}{\pi}}$ and $\sigma^{2}\left(1-\frac{2 \delta^{2}}{\pi}\right)$, respectively, where $\delta \equiv$ $\beta / \sqrt{1+\beta^{2}}$. Clearly,

$f(x ; \mu, \sigma, \beta) \rightarrow \frac{1}{\sigma} \phi\left(\frac{x-\mu}{\sigma}\right)$

as $\beta \rightarrow 0$.

In our simulations, we will consider data generating processes where the outcome is generated as

$Y=T V_{G}+(1-T) V_{H}$,

where $T$ is a latent binary variable, and $V_{G} \sim G$ and $V_{H} \sim H$. Both error distributions $G$ and $H$ are skewed-normal distributions with parameters $\mu_{G}, \sigma_{G}, \beta_{G}$ and $\mu_{H}, \sigma_{H}, \beta_{H}$, respectively.

From Capitanio (2010) it follows that Assumption 8 holds if $G$ is right-skewed and $H$ is left-skewed. We will consider designs where $\beta_{G}>0$ and $\beta_{H}<0$ to verify our asymptotics.

When $\beta_{G}=\beta_{H}=0$, (3.1) collapses to a standard location model with normal errors:

$Y=\left(\mu_{G}-\mu_{H}\right) T+V, \quad V \sim \mathcal{N}\left(0, \sigma_{G}^{2}+\sigma_{H}^{2}\right)$.

The identifying tail condition in Assumption 3 still holds if $\mu_{G}>\mu_{H}$, and our estimators remain consistent. However, Assumption 8 now fails and so we may expect poor inference in this design.

In our experiments, we generate a binary $X$ with $\mathbb{P}(X=1)=\frac{1}{2}$ and fix conditional probabilities as

$$
\begin{aligned}
& \mathbb{P}(T=0 \mid X=0)=\frac{3}{4}, \mathbb{P}(T=1 \mid X=0)=\frac{1}{4}, \\
& \mathbb{P}(T=1 \mid X=1)=\frac{1}{4}, \mathbb{P}(T=1 \mid X=1)=\frac{3}{4} .
\end{aligned}
$$

We present results for data generating processes, where $\mu_{G}=\mu=-\mu_{H}$ and $\beta_{G}=\beta=-\beta_{H}$. We use the designs $\mu=0$ and $\beta \in\{2.5,5\}$ to evaluate the adequacy of our asymptotic arguments for small-sample inference. We also look at the performance of our estimators when $\mu \in\{.5,1\}$ and $\beta=0$, which yields the Gaussian location model in (3.2). We fix $\sigma_{G}=\sigma_{H}=1$ throughout. For each of these designs, we consider choices of the empirical quantiles as

$l_{n_{x}}=C\left(n_{x} \ln \ln n_{x}\right)^{6 / 10}, \quad \kappa_{n_{x}}=C\left(n_{x} \ln \ln n_{x}\right)^{6 / 10}$ 
for several choices of the constant $C$. All of these choices are in line with our asymptotic arguments. The larger the constant $C$ the more conservative the choice of intermediate quantile,

$q_{\ell} \equiv \frac{l_{n_{x}}}{n_{x}}, \quad q_{r} \equiv \frac{n_{x}-\kappa_{n_{x}}}{n_{x}}$

for a given sample size.

We run experiments for sample sizes $n \in\{500 ; 1,000,2,500 ; 5,000 ; 10,000$; $25,000\}$. We report (the average over the replications of) $q_{\ell}$ and $q_{r}$ along with the estimation results to get an idea of how far in the tails of the component distributions we are going to obtain the results. A data-driven determination of the constant $C$ is challenging and is left for future research. For space considerations, we report only a subset of the results here. The full set of simulation results is available in the working paper version of this paper (Jochmans, Henry, and Salanié, 2014).

Tables 1 and 2 report the results for the mixing proportions $\lambda(0)$ and $\lambda(1)$. Each table contains the bias, standard deviation (SD), ratio of the (average over the replications of the) estimated standard error to the standard deviation (SE/SD), and the coverage of $95 \%$ confidence intervals (CI95) for $n \in\{1,000,10,000\}$. All these statistics were computed from 10,000 Monte Carlo replications. Table 1 reports results for the simulation design with $\mu=0, \beta=5$ for $C \in\{.5,1,1.5\}$, so as to evaluate the impact of the choice of this tuning parameter on the results. This impact was similar in all other designs and so, for these designs, we present only results for one choice of $C$. The constant $C$ was fixed to .5 for all designs except for the pure location model with $\mu=.5$ and $\beta=0$, where, for practical reasons, we use $C=.75 .^{4}$ These results are bundled in Table 2 .

The results in Table 1 support our asymptotic theory. For all choices of the tuning parameter $C$, the bias and standard deviation shrink to zero as $n \uparrow+\infty$; and

TABLE 1. Mixing proportions

\begin{tabular}{|c|c|c|c|c|c|c|c|c|c|c|}
\hline \multirow[b]{2}{*}{$n$} & \multirow[b]{2}{*}{$q_{\ell}$} & \multirow[b]{2}{*}{$q_{r}$} & \multicolumn{2}{|c|}{ BIAS } & \multicolumn{2}{|c|}{ SD } & \multicolumn{2}{|c|}{ SE/SD } & \multicolumn{2}{|c|}{ CI95 } \\
\hline & & & $\lambda_{n}(0)$ & $\lambda_{n}(1)$ & $\lambda_{n}(0)$ & $\lambda_{n}(1)$ & $\lambda_{n}(0)$ & $\lambda_{n}(1)$ & $\lambda_{n}(0)$ & $\lambda_{n}(1)$ \\
\hline \multicolumn{11}{|c|}{$C=.5$} \\
\hline 1,000 & .059 & .940 & .0060 & -.0059 & .0693 & .0701 & 1.0554 & 1.0392 & .9688 & .9682 \\
\hline 10,000 & .026 & .974 & .0012 & -.0011 & .0328 & .0325 & 1.0106 & 1.0213 & .9560 & .9572 \\
\hline \multicolumn{11}{|c|}{$C=1$} \\
\hline 1,000 & .120 & .880 & .0024 & -.0035 & .0439 & .0446 & 1.1358 & 1.1220 & .9764 & .9752 \\
\hline 10,000 & .052 & .947 & .0007 & -.0003 & .0225 & .0222 & 1.0360 & 1.0519 & .9566 & .9616 \\
\hline \multicolumn{11}{|c|}{$C=1.5$} \\
\hline 1,000 & .179 & .821 & .0046 & -.0037 & .0316 & .0315 & 1.2931 & 1.2933 & .9944 & .9920 \\
\hline 10,000 & .078 & .922 & .0002 & -.0010 & .0175 & .0174 & 1.0873 & 1.0962 & .9646 & .9710 \\
\hline
\end{tabular}


TABLE 2. Mixing proportions (cont'd)

\begin{tabular}{|c|c|c|c|c|c|c|c|c|c|c|}
\hline \multirow[b]{2}{*}{$n$} & \multirow[b]{2}{*}{$q_{\ell}$} & \multirow[b]{2}{*}{$q_{r}$} & \multicolumn{2}{|c|}{ BIAS } & \multicolumn{2}{|c|}{ SD } & \multicolumn{2}{|c|}{$\mathrm{SE} / \mathrm{SD}$} & \multicolumn{2}{|c|}{$\mathrm{CI} 95$} \\
\hline & & & $\lambda_{n}(0)$ & $\lambda_{n}(1)$ & $\lambda_{n}(0)$ & $\lambda_{n}(1)$ & $\lambda_{n}(0)$ & $\lambda_{n}(1)$ & $\lambda_{n}(0)$ & $\lambda_{n}(1)$ \\
\hline \multicolumn{11}{|c|}{$\mu=0$ and $\beta=2.5$} \\
\hline 1,000 & .059 & .940 & .0066 & -.0072 & .0722 & .0718 & 1.0151 & 1.0194 & .9646 & .9652 \\
\hline 10,000 & .026 & .974 & .0012 & -.0015 & .0323 & .0326 & 1.0287 & 1.0193 & .9548 & .9626 \\
\hline \multicolumn{11}{|c|}{$\mu=1$ and $\beta=0$} \\
\hline 1,000 & .059 & .940 & .0144 & -.0164 & .0720 & .0728 & 1.0589 & 1.0518 & .9807 & .9810 \\
\hline 10,000 & .026 & .974 & .0050 & -.0048 & .0327 & .0324 & 1.0344 & 1.0449 & .9614 & .9622 \\
\hline \multicolumn{11}{|c|}{$\mu=.5$ and $\beta=0$} \\
\hline 1,000 & .090 & .910 & .0994 & -.1017 & .0842 & .0855 & 1.1677 & 1.1599 & .9416 & .9406 \\
\hline 10,000 & .039 & .961 & .0671 & -.0671 & .0358 & .0352 & 1.0815 & 1.0973 & .6244 & .6286 \\
\hline
\end{tabular}

the bias is small relative to the standard error. Furthermore, SE/SD $\rightarrow 1$ and the coverage rates of the confidence intervals are close to .95 in large samples. The variability of the point estimates is somewhat overestimated when $n$ is very small and $C$ is chosen conservatively. Together with the relatively small bias, this implies that confidence intervals are slightly conservative. For $C=.5$, coverage rates are close to .95 , even for the smallest samples considered, and for all $C$, the coverage rates move fairly quickly toward .95 as $n$ increases. The same conclusions hold for the design with $\mu=0$ and $\beta=2.5$ (first block of Table 2).

Now turn to the results for the pure location model with Gaussian errors $(\beta=0)$ in Table 2, where the tail conditions of Assumption 8 fail. The difference between the two designs is the distance between the component distributions (governed by $\mu$ ). When $\mu=1, G$ is centered at 1 while $H$ is centered at -1 , so that $\mu_{G}-\mu_{H}=2$. When $\mu=1 / 2, G$ and $H$ are closer to each other: $\mu_{G}-\mu_{H}=1$. In the first of these designs, the bias in the point estimates is somewhat larger than in the skewed designs. Nonetheless, the bias is still small relative to the standard deviation. Furthermore, the coverage of the confidence intervals displays a similar pattern as before, and is excellent when $n$ is not too small. When we move to the second design, the bias increases further. The bias still shrinks to zero as $n$ grows, confirming that our estimator remains consistent. However, the bias is not negligible relative to the standard deviation; the coverage of the confidence intervals deteriorates as $n$ grows, and inference becomes unreliable.

We next turn to the results for the component distributions. For clarity, we present the results by means of a series of plots. We provide results for $n=1,000$ for the skewed designs $\mu=0, \beta=5$ and $\mu=0, \beta=2.5$ in Figure 1 and for the symmetric designs $\mu=1, \beta=0$ and $\mu=0.5, \beta=0$ in Figure 2. Results for $G_{n}$ are in the left-side plots. Results for $H_{n}$ are in the right-side plots. Each plot contains the mean of the point estimates (solid red lines) and the mean of $95 \%$ confidence 

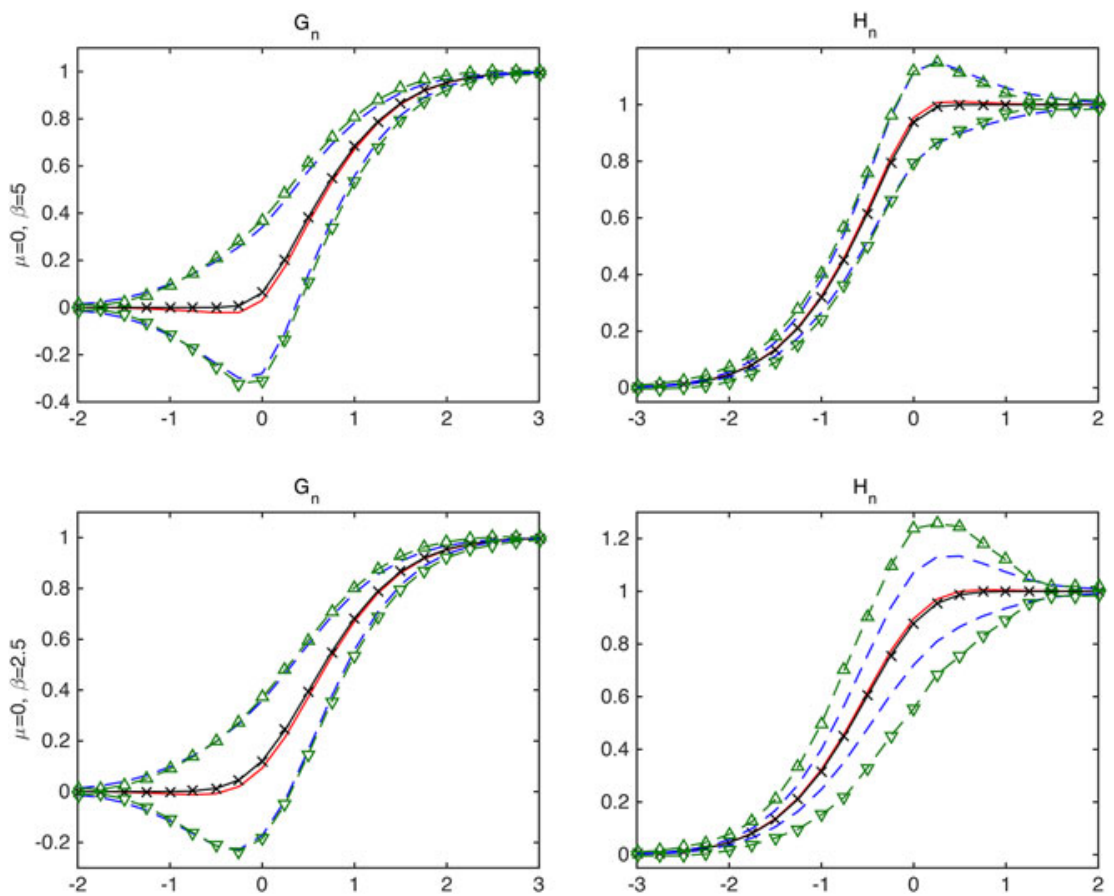

FIGURE 1. Simulation results for $G_{n}$ (left) and $H_{n}$ (right) for design $\mu=0, \beta=5$ (top) and design $\mu=0, \beta=2.5$ (bottom). Each plot contains the mean of the point estimator (solid red line) and the mean of the estimated $95 \%$ confidence bands (dashed blue lines), along with the true curve (solid black line, marked x) and 95\% confidence bands constructed using the Monte Carlo standard deviation (dashed green lines, upper band marked $\triangle$ and lower band marked $\nabla$ ).

bounds constructed around it using a plug-in estimator of the asymptotic variance in Theorem 5 (dashed blue lines). Each plot also contains the true component distribution (solid black lines, marked $\mathrm{x}$ ) and the mean of $95 \%$ confidence bounds constructed around the point estimator using the empirical standard deviation over the Monte Carlo replications (dashed green lines, upper band marked $\triangle$, lower band marked $\nabla$ ). We vary the range of the vertical axis across the plots in a given figure to enhance visibility.

The plots in Figure 1 again confirm our asymptotics. The bias in the point estimators is small across all plots. The asymptotic theory mostly does a good job in capturing the small-sample variability of the point estimators although, when $n$ is small, the standard errors are somewhat too small. In our designs, this underestimation is more severe for $H_{n}$ than for $G_{n}$, as is apparent from inspection of the lower-right plot in the figure. Inspection of the full set of results (not reported here) shows that this underestimation vanishes as $n$ grows, again confirming our asymptotic theory. 

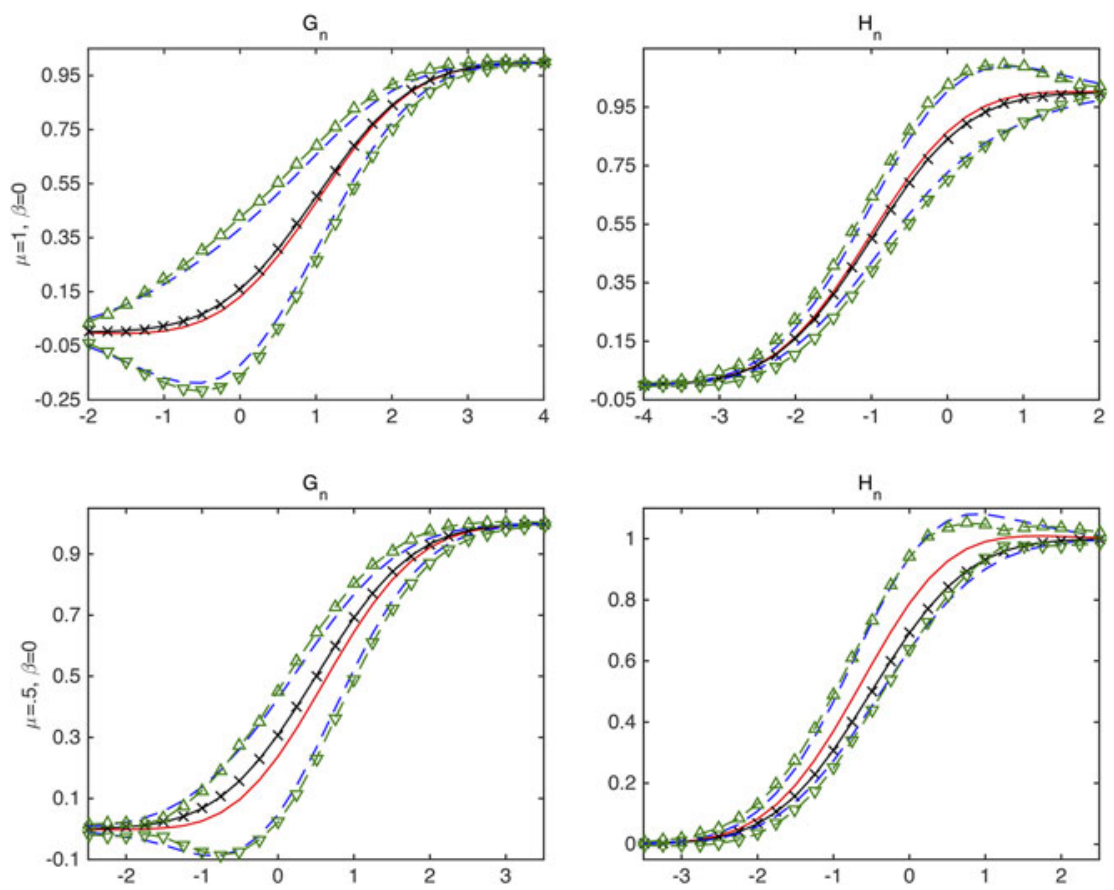

FigURE 2. Simulation results for $G_{n}$ (left) and $H_{n}$ (right) for design $\mu=1, \beta=0$ (top) and design $\mu=0.5, \beta=0$ (bottom). Each plot contains the mean of the point estimator (solid red line) and the mean of the estimated $95 \%$ confidence bands (dashed blue lines), along with the true curve (solid black line, marked x) and 95\% confidence bands constructed using the Monte Carlo standard deviation (dashed green lines, upper band marked $\triangle$ and lower band marked $\nabla$ ).

The results in Figure 2 for the Gaussian location model are in line with our findings concerning the mixing proportions. In the design where $\mu_{G}-\mu_{H}=2$ (upper two plots), our estimators do well in spite of Assumption 8 not holding. When the $\mu_{G}-\mu_{H}=1$ (lower two plots), however, the asymptotic bias in $G_{n}$ and $H_{n}$ becomes visible. While the variability of the point estimates is correctly captured by our asymptotic-variance estimator, the confidence bounds settle around an incorrect curve.

\section{Concluding remarks}

We conducted most of our analysis with a mixture of two components. However, some of our results would extend to a version of (1.1) with a larger number of components. Suppose that the mixture has $J$ irreducible components, as in

$F(y \mid x)=\sum_{j=1}^{J} \lambda_{j}(x) G_{j}(y)$, 
in obvious notation. Henry et al. (2014) showed that the mixture components and mixing proportions are only identified up to $J(J-1)$ inequality-constrained real parameters in general.

Tail dominance restrictions can still be quite powerful. Take $J=3$ for instance, and assume that $G_{1}$ dominates in the left tail and $G_{3}$ dominates in the right tail. Then it is easy to adapt the proof of Theorem 1 to prove that the behavior of $F(y \mid x)$ in the left tail identifies the function $\lambda_{1}$ up to a multiplicative constant, and that the behavior of $F(y \mid x)$ in the right tail identifies the function $\lambda_{3}$ up to another multiplicative constant. Imposing the values of the mixing proportions at one particular value of $x$ would be enough to point identify all elements of the model, for instance; and it would be easy to adapt our estimators and tests to such a setting. Whether such additonal restrictions are plausible is, of course, highly model-dependent.

\section{NOTES}

1. We omit conditioning variables throughout. The identification analysis extends straightforwardly. In principle, the distribution theory could be extended by using local empirical process results along the lines of Einmahl and Mason (1997). We postpone a detailed investigation into such an extension to future work.

2. Note that irreducibility rules out the possibility of achieving identification of $G$ and $H$ via an identification-at-infinity argument, as in Heckman (1990) and Andrews and Schafgans (1998) for instance.

3. The expression for $\lambda\left(x^{\prime}\right)$ in (1.3) also holds for any $x^{\prime \prime}$. This invariance cannot fruitfully be exploited to test the tail restrictions of Assumption 3, however, as the right-hand side expression in (1.3) is independent of the value $x^{\prime \prime}$ even when Assumption 3 fails.

4. In this design, there is a small probability that either $q_{\ell}=0$ or $q_{r}=1$ when $C=.5$ and $n$ is small. This shows up in simulations with a large number of replications, as is the case here. The slightly more conservative choice of $C=.75$ avoids this issue.

\section{REFERENCES}

Acemoglu, D., V. Carvalho, A. Ozdaglar, \& A. Tabaz-Salehi (2012) The network origins of aggregate fluctuations. Econometrica 80(5), 1977-2016.

Allman, E.S., C. Matias, \& J.A. Rhodes (2009) Identifiability of parameters in latent structure models with many observed variables. Annals of Statistics 37, 3099-3132.

Andrews, D.W.K. \& M.M.A. Schafgans (1998) Semiparametric estimation of the intercept of a sample selection model. Review of Economic Studies 65, 497-517.

Arkolakis, C., A. Costinot, \& A. Rodriguez-Clare (2012) New trade models, same old gains? American Economic Review 102, 94-130.

Atkinson, A.B., T. Piketty, \& E. Saez (2011) Top incomes in the long run of history. Journal of Economic Literature 49, 3-71.

Azzalini, A. (1985) A class of distributions which includes the normal ones. Scandinavian Journal of Statistics 12, 171-178.

Bollinger, C.R. (1996) Bounding mean regressions when a binary regressor is mismeasured. Journal of Econometrics 73, 387-399.

Bonhomme, S., K. Jochmans, \& J.-M. Robin (2016) Estimating multivariate latent-structure models. Annals of Statistics, 44, 540-563.

Bonhomme, S., K. Jochmans, \& J.-M. Robin (2016) Nonparametric estimation of finite mixtures from repeated measurements. Journal of the Royal Statistical Society, Series B 78, 211-229. 
Bordes, L., S. Mottelet, \& P. Vandekerkhove (2006) Semiparametric estimation of a two-component mixture model. Annals of Statistics 34, 1204-1232.

Capitanio, A. (2010) On the approximation of the tail probability of the scalar skew-normal distribution. METRON 68, 299-308.

Carroll, R.J., D. Ruppert, L.A. Stefanski, \& C. Crainiceanu (2006) Measurement Error in Nonlinear Models: A Modern Perspective. Chapman and Hall, CRC Press.

D'Haultfœuille, X. \& P. Février (2015) Identification of mixture models using support variations. Journal of Econometrics 189, 70-82.

D'Haultfœuille, X. \& A. Maurel (2013) Another look at identification at infinity of sample selection models. Econometric Theory 29, 213-224.

Einmahl, J. (1992) Limit theorems for tail processes with application to intermediate quantile estimation. Journal of Statistical Planning and Inference 32, 137-145.

Einmahl, U. \& D. Mason (1997) Gaussian approximation of local empirical processes indexed by functions. Probability Theory and Related Fields 107, 283-311.

Frisch, R. (1934) Statistical confluence analysis by means of complete regression systems. Technical Report 5, University of Oslo, Economics Institute, Oslo, Norway.

Gabaix, X. (2009) Power laws in economics and finance. Annual Review of Economics 1, 255-294.

Gassiat, E. \& J. Rousseau (2016) Nonparametric finite translation hidden Markov models and extensions. Bernoulli 22, 193-212.

Ghysels, E., A. Harvey, \& E. Renault (1996) Stochastic volatility. In G.S. Maddala \& C.R. Rao (eds.), Handbook of Statistics Volume 14: Statistical Methods in Finance. Elsevier.

Hall, P. \& X.-H. Zhou (2003) Nonparametric identification of component distributions in a multivariate mixture. Annals of Statistics 31, 201-224.

Hamilton, J.D. (1989) A new approach to the analysis of nonstationary times series and the business cycle. Econometrica 57, 357-384.

Heckman, J.J. (1974) Shadow prices, market wages, and labor supply. Econometrica 42, 679-694.

Heckman, J.J. (1990) Varieties of selection bias. American Economic Review 80, 313-318.

Henry, M., Y. Kitamura, \& B. Salanié (2010) Identifying Finite Mixtures in Econometric Models. Cowles Foundation Discussion paper 1767.

Henry, M., Y. Kitamura, \& B. Salanié (2014) Partial identification of finite mixtures in econometric models. Quantitative Economics 5, 123-144.

Hu, Y. \& S.M. Schennach (2008) Instrumental variable treatment of nonclassical measurement error models. Econometrica 76, 195-216.

Jochmans, K., M. Henry, \& B. Salanié (2014) Inference on mixtures under tail restrictions. Discussion paper No. 2014-01, Department of Economics, Sciences Po.

Kasahara, H. \& K. Shimotsu (2009) Nonparametric identification of finite mixture models of dynamic discrete choices. Econometrica 77, 135-175.

Khan, S. \& E. Tamer (2010) Irregular identification, support conditions and inverse weight estimation. Econometrica 78, 2021-2042.

Lewbel, A. (2007) Estimation of average treatment effects with misclassification. Econometrica 75, 537-551.

Mahajan, A. (2006) Identification and estimation of regression models with misclassification. Econometrica 74, 631-665.

Schwarz, M. \& S. Van Bellegem (2010) Consistent density deconvolution under partially known error distribution. Statistics and Probability Letters 80, 236-241.

Shimer, R. L. Smith (2000) Assortative matching and search. Econometrica 68, 343-369. 